\title{
Hospital Readmission Prediction using Discriminative patterns
}

\author{
Sea Jung Im \\ School of Computer Science \\ Queensland University of Technology \\ Brisbane, Australia \\ seajung.im@hdr.qut.edu.au \\ Ann Bonner \\ School of Nursing and Midwifery \\ Griffith University \\ Brisbane, Australia \\ a.bonner@griffith.edu.au
}

\author{
Yue Xu \\ School of Computer Science \\ Queensland University of Technolog \\ Brisbane, Australia \\ yue.xu@qut.edu.au \\ Helen Healy \\ Metro North Hospital \& Health Service \\ Queensland Health \\ Brisbane, Australia \\ Helen.Healy@health.qld.gov.au
}

\author{
Jason Watson \\ School of Information Systems \\ Queensland University of Technology \\ Brisbane, Australia \\ ja.watson@qut.edu.au \\ Wendy Hoy \\ Faculty of Medicine \\ The University of Queenland \\ Brisbane, Australia \\ w.hoy@uq.edu.au
}

\begin{abstract}
Avoidable hospital readmission is problematic as it increases the burden on healthcare systems. leads to a shortage of hospital beds and impacts on the costs of healthcare. Various machine learning algorithms have been applied to predict patient readmissions. However, existing literature has only focused on individual features of health conditions without consideration of associations between features. This paper proposes discriminative pattern-based features as a technique to improve readmission prediction. First, discriminative patterns that occur disproportionately between two classes: readmission and non-readmission, were generated based on hospital electronic health records. Second, the patterns were fed as features into a classification model for readmission prediction. We have evaluated these discriminative pattern-based features in three datasets: diabetes, chronic kidney disease and all diseases. Experiments with each dataset showed that the features of chronic disease cohorts have less differences between the readmission and the non-readmission classes than the alldiseases cohort. Our proposed pattern-based model improved the prediction performance in terms of AUC (Area Under the receiver operating characteristic curve) by about $12 \%$ compared with the baseline models for the all-disease cohort, however it showed little improvement for either the diabetes or chronic kidney disease datasets.
\end{abstract}

Keywords-Hospital readmission, pattern mining, discriminative patterns

\section{INTRODUCTION}

A hospital readmission happens when a patient returns to the same hospital or another hospital within a certain time frame after discharge from the initial admission. Excessive readmissions use up resources and are a significant cost for hospitals. For example, readmissions within 30 days accounted for $20 \%$ of all admissions and cost about $\$ 17$ million in 2004, the United State [1]. Now, many countries consider hospital readmission rates as a measurement of the quality of hospital services; therefore, reducing patient readmissions in hospitals is an issue of great concern to funding bodies.

Preventing readmission can be achieved by understanding key factors leading to the hospital readmission and predicting who is likely to be readmitted after discharge. When a patient is identified as at high risk, clinicians can provide additional interventions to reduce the chance of readmission, which provide patients with a higher quality of service and can avoid unnecessary expenditure. Thus, it has become important to identify who is likely to be readmitted. Due to the widespread adoption of electronic health records systems (EHR) around the world, researchers have a keen interest in using machine learning algorithms to produce prediction models which are well known to have the capability to handle complex data.

Various machine learning algorithms have been applied to predict the likelihood of patients' readmission and to discover leading factors for readmissions. Logistic regression has been the most widely applied algorithm to predict readmissions $[2,3]$. In recent years, more complicated machine learning techniques such as random forests, and gradient boosting methods, have been implemented to improve the performance of readmission prediction [4]. Most of the prediction models utilise demographic, historical and medical information about patients for prediction. However, these models limit their approaches focusing on individual features not considering correlations between them. Considering that many features such as patients' demographic features, medications, diagnoses, procedures are related to each other, it is reasonable to take relationships between these features into account when predicting readmission.

To address this issue, we propose a new type of discriminative patterns which can be generated from an EHR dataset. These patterns can be used as features to represent patients for prediction. In the context of readmission prediction, patients can be classified into two classes, readmission and no-readmission, based on whether they have a readmission history or not. Pattern mining is a typical data mining technique to discover associations between individual features in large transactional datasets. A pattern is a set of individual features which are associated with each other. The discriminative patterns are a set of specific patterns which occur disproportionately in the two patient classes. We believe discriminative patterns would differentiate the two patient classes more clearly than individual features by utilising the associations between features. Several methods have been suggested to find discriminative patterns including ratio or difference of frequency $[5,22]$. In this study, we take both frequency and frequency differences into consideration to find strong discriminative patterns.

The main contributions of this study are summarised as follows: 1) to propose a new type of discriminative pattern to differentiate objects in two classes for readmission prediction 2) to build a classification model to predict readmission by using the discriminative patterns as features, and 3) conduct experiments to evaluate the prediction performance of the proposed models. 


\section{RELATED WORKS}

Feature selection is one of the critical steps for building classification models. In some previous studies, features for readmission prediction were mainly selected from patients' EHR data based on advice from domain experts using their medical knowledge and experience. Another popularly used method for feature selection is the univariate feature selection that chooses features based on statistical significance measured by chi-square, information gain, or Odds ratio [6-9]. Strack, DeShazo, Gennings et al. Reference [6] proposed LACE (Length of stay, Acuity of the encounter, Comorbidity index score, and Emergency department use in six months before the admission) for hospital readmission prediction. These are the most widely used features for predicting readmission. Cui, Wang, Wang et al. [9] compared various statistical measures and demonstrated that chi-square outperforms over other methods on accuracy for readmission prediction. These authors recommend that the selected features are 'the number of visits in the preceding year', 'diagnostic information', 'discharge disposition', 'number of procedures during the encounter', and 'time in hospital' and so on. Bradford, Shah, Shane et al. [8] compared the characteristics of readmission group and no-readmission group via chi-square and proposed 5 factors contributing to 30-day readmission which include: 'employment status', 'disable status', 'emergency department visit in the past 90 days', 'longer than 5 days of the length of stay during the visit', and 'a serum urea level $>45 \mathrm{mg} / \mathrm{dL}$ '.

Discriminative pattern mining has also been proposed to detect differences between classes and improve the performance of classification [5, 21, 22]. SupMaxK proposed in [22] is an algorithm to generate patterns that have high support in one class and low support in the other class. Authors from [5] proposed C-Motif algorithm to generate a pattern type called Conditional Phosphorylation Motif which is based on the relative support ratio or support odds ratio, and also a local significance measure. The local significance measure evaluates the significances of sub-items of a pattern so that the algorithm chooses patterns with sub-items having local significance greater than the defined threshold.

\section{METHODS}

\section{A. Problem Statements}

In this section, we explain the necessity of choosing discriminative patterns as features for classification-based readmission prediction. As mentioned above, many attempts to find the best features for classification have been conducted. However, these mainly focus on the correlation between individual features and readmission/nonreadmission. However, few studies have considered associations between features for classification. Frequent pattern mining has been widely adopted and proved that it can enhance the performance of classification by discovering the relationships between features [10]. Most pattern mining algorithms are to generate frequent patterns which occur frequently in the whole dataset. These frequent patterns are certainly important because frequent occurrence means that it has a significant role in a dataset. However, frequent patterns are useless if it exists in two classes with a similar proportion. These patterns cannot represent any class even though it is frequent because they cannot differentiate the two classes. Thus, we propose the discriminative patterns to enhance the characteristics of each class.
For further illustration, take Fig. 1 as an example. The sample dataset consists of two classes: positive class (+) and negative class (-). Each class has 10 objects each of which contains some of the 10 features $x_{1}, \ldots, x_{10}$. For the first object, it is in a positive class and contains two features $\left\{x_{4}, x_{5}\right\}$. Several patterns can be generated in Fig. 1. We consider a pattern frequent in its class if it occurs in more than half of the objects of that class. We have two frequent item sets: $I_{1}=\left\{x_{1}, x_{2}, x_{4}, x_{5}\right\}$ for the positive class and $I_{2}=$ $\left\{x_{4}, x_{5}, x_{6}, x_{7}\right\}$ for the negative class. Then, frequent patterns for size 2 for the positive class include $P 1=\left\{x_{1}, x_{2}\right\}$, and $P 2=\left\{x_{4}, x_{5}\right\}$, while for the negative class is $P 2=\left\{x_{4}, x_{5}\right\}$ and $P 3=\left\{x_{6}, x_{7}\right\}$ as the number of occurrence of these patterns in each class is greater than 5. As shown in Fig. 1, $P 1$ only appears in the positive class whereas $P 3$ exists only in the negative class, which means it has discriminative features. In contrast, as $P 2$ appears in both classes with the same proportion, it hardly represents any class. Thus, even though $P 2$ is frequent, it is not a discriminative pattern. Whether a pattern can be discriminative or not depends on the threshold of how much differences between the two classes should be. Once the threshold is set, discriminative patterns will be selected by comparing the threshold with the differences of frequency between two classes. For $P 1$, the number of objects in the positive class is 7 , while 0 in the negative class. As a result, the difference is 7 . If the threshold is set as 5 , it will be a discriminative pattern.

\begin{tabular}{|c|c|c|c|c|c|c|c|c|}
\hline ID & Class & $\boldsymbol{x}_{\mathbf{1}}$ & $\boldsymbol{x}_{\mathbf{2}}$ & $\boldsymbol{x}_{\mathbf{3}}$ & $\boldsymbol{x}_{\mathbf{4}}$ & $\boldsymbol{x}_{\mathbf{5}}$ & $\boldsymbol{x}_{\mathbf{6}}$ & $\boldsymbol{x}_{\mathbf{7}}$ \\
\hline 1 & + & & & & & & & \\
\hline 2 & + & & & & & & & \\
\hline 3 & + & & & & & & & \\
\hline 4 & + & & & & & & & \\
\hline 5 & + & & & & & & & \\
\hline 6 & + & & & & & & & \\
\hline 7 & + & & & & & & & \\
\hline 8 & + & & & & & & & \\
\hline 9 & + & & & & & & & \\
\hline 10 & + & & & & & & & \\
\hline 11 & - & & & & & & & \\
\hline 12 & - & & & & & & & \\
\hline 13 & - & & & & & & & \\
\hline 14 & - & & & & & & & \\
\hline 15 & - & & & & & & & \\
\hline 16 & - & & & & & & \\
\hline 17 & - & & & & & & \\
\hline 18 & - & & & & & & & \\
\hline 19 & - & & & & & & & \\
\hline 20 & - & & & & & & & \\
\hline
\end{tabular}

Figure 1 sample of the dataset. There are two classes each has 10 objects. Rows indicate objects, and columns indicate individual features. The two classes can be clearly differentiated from each other by discriminative patterns $\mathrm{p} 1=\left\{x_{1}, x_{2}\right\}$ and $\mathrm{p} 3=\left\{x_{6}, x_{7}\right\}$

\section{B. Discriminative patterns}

Let $D$ be a data set with two classes. Each object in $D$ is represented by a set of features or attributes. $D$ can be divided into two subsets based on classes, $D_{1}$ contains objects representing positive class (e.g., readmission class) and $D_{2}$ 
contains objects representing negative class (e.g., nonreadmission class), thus $D=D_{1}, \cup D_{2}, D_{1}, \cap D_{2}=\emptyset$. For a pattern, $p=\left\{x_{1}, x_{2}, x_{3}, \ldots \ldots, x_{n}\right\}, x_{i}$ is a feature or attribute in $D$, the relative support or simply support of $p$ over $D_{1}$ is:

$$
\sup \left(p, D_{1}\right)=\frac{\text { number of objects in } D_{1} \text { which contain } p}{\text { total number of objects in } D_{1}}
$$

A pattern $\mathrm{p}$ is frequent in $D_{1}$ when $\sup \left(p, D_{1}\right)$ is no less than a given threshold $\left(0<\theta_{\text {sup }}<1\right)$. After generating all frequent patterns from each class, we now compare the support of these patterns between two data sets and calculate differences. The absolute difference of support of the pattern $p$ in terms of $D_{1}$ and $D_{2}$ is defined below, where $D_{1}$ is considered as a base class:

$$
A S D\left(p, D_{1}\right)=\sup \left(p, D_{1}\right)-\sup \left(p, D_{2}\right)
$$

The ratio of absolute difference of support over base class, called 'relative support difference', is defined as

$$
\operatorname{RSD}\left(p, D_{1}\right)=\frac{A S D\left(p, D_{1}\right)}{\sup \left(p, D_{1}\right)}
$$

In this research, the relative support difference will be used to measure the discriminative power of patterns. If $R S D\left(p, D_{1}, D_{2}\right) \geq \theta_{r s d}, \theta_{r s d}$ is the minimum relative difference threshold, $p$ is considered as a discriminative pattern. Discriminative patterns from both classes will be selected as input features. The parameters required in this study are support threshold $\theta_{\text {sup }}$ and the relative support difference threshold $\theta_{\text {rsd }}$. The discriminative patterns are required to be $\sup \left(p, D_{1}\right) \geq \theta_{\text {sup }}$ and $\operatorname{RSD}\left(p, D_{1}, D_{2}\right) \geq$ $\theta_{\text {rsd }}$. Additionally, we propose a new measure, called discrimination score to select the best discriminative patterns by taking into account both support and the relative support difference of patterns. It is calculated as a linear combination of the two parts with weight $w$ to determine the importance of each of them. Setting $w=0.5$ indicates that we consider the support and the relative support difference with the same weight.

$$
\begin{gathered}
\operatorname{DCS} \operatorname{core}\left(p, D_{1}\right)=w * \sup _{(0<w}\left(p, D_{1}\right)+(1-w) * R S D\left(p, D_{1}\right) \\
(0<w<1)
\end{gathered}
$$

\section{Algorithm}

The algorithm of generating discriminative patterns for predicting patient readmission is presented in Algorithm 1. To begin with, we divided the original dataset into two datasets based on its classes: readmission class and no-readmission class (Line1). Then, frequent patterns of up to size $\mathrm{k}$ are generated from each class by applying the Apriori pruning algorithm (Line2 3). Next, we will calculate the relative support difference by comparing the support value of patterns from each class (Line 5) and the discriminative score with predefined weight value $w$ (Line 6). When a pattern has the relative support difference and the discriminative score that is greater than each threshold, it will be selected as a discriminative pattern (Line 7 8). In the end, a set of discriminative patterns from each class will be generated.

\section{EXPERIMENTS AND EVALUATION}

We evaluated the proposed discriminative patterns by comparing the performance of readmission prediction models against existing algorithms.

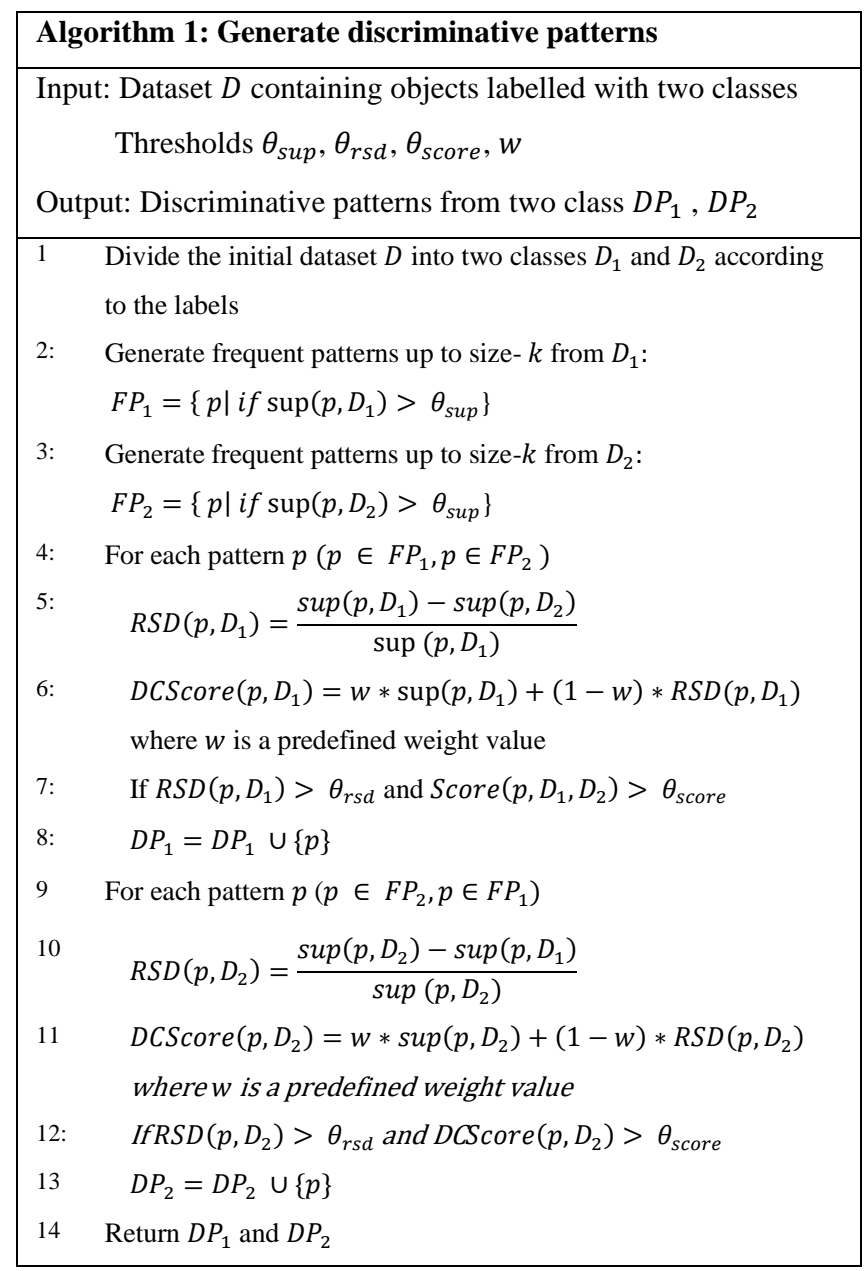

\section{A. Datasets}

We used 3 datasets in the evaluation: diabetes, chronic kidney disease, and all-diseases cohorts.

\section{1) Diabetes dataset}

It contains 10 years (1999-2008) of medical data at 130 hospitals from the United States. It includes 53 features representing patients with at least diabetes among three diagnoses [16].

\section{2) $\mathrm{MIMIC-III}$}

This dataset is from the Beth Israel Deaconess Medical Centre in Boston, Massachusetts, which is publicly available for research purposes. It stores extensive data about patients with all diseases including demographics, diagnoses, medications, procedures and lab tests between 2001 and 2012 [17].

\section{3) CKD.QLD Registry}

It contains hospital admission data for a group of patients with chronic kidney diseases (CKD) receiving renal specialty care in the public health system in Queensland, Australia between 2011 and 2018 [18]. Admissions with dialysis codes such as 'Z491', Z492', 'Z490', are removed as they are not unplanned [24]. 
In the experiment, an admission is considered as a readmission if a patient returns to a hospital within 30 days after being discharged with no planning. When patients have multiple admissions in the datasets, we calculate the number of days from the last discharge date and if the admission occurs within 30 days, then this is labelled as 1, readmission, otherwise 0. Diagnosiss codes (ICD-9 for diabetes and MIMIC-III datasets, ICD-10-AM for CKD.QLD dataset) are essential individual features in the datasets. There are 954, 6984, 5455 diagnosis codes occurring in diabetes, MINIC-III, and CKD.QLD datasets, respectively. In fact, many diagnosis codes belong to the same category. Based on the Elixhauser index comorbidities [19,23], the diagnosis codes were firstly converted into 31 groups. The diagnosis codes that are not included in Elixhauser comorbidities are assigned to the fourth-digit categories in ICD-9 classification or three-digit categories in ICD-10-AM. Finally, all continuous features were discretised into categorical features, making the total number of features 64, 56, and 63 for diabetes, CKD.QLD and MIMIC-III datasets respectively.

A summary of each dataset is shown in Table 1. The readmission rates of three datasets are $11.3 \%, 22.4 \%$ and $5.4 \%$ for diabetes, CKD.QLD and MIMIC III datasets respectively, which means they are very imbalanced. Diabetes and CKD, which are both known as chronic diseases have higher readmission rates than the all-disease cohort, MIMIC III.

\begin{tabular}{|l|r|r|r|}
\hline & Diabetes & CKD.QLD & MIMIC III \\
\hline Number of patients & 59,561 & 6,058 & 41,225 \\
\hline Number of admissions & 82,894 & 28,484 & 51,271 \\
\hline Number of diagnosis codes & 954 & 5455 & 6984 \\
\hline Rate of Readmission & $11.3 \%$ & $22.4 \%$ & $5.4 \%$ \\
\hline
\end{tabular}

Table 1 Summary of three datasets

Table 2 shows the relative support difference of some features including 11 of diagnoses groups between two classes in the datasets. The average relative support differences of all features are $0.15,0.11$, and 0.27 for diabetes, CKD.QLD and MIMIC-III datasets respectively. The MIMIC-III dataset was found to have a relatively higher average relative support difference compared to the other two datasets; however, we can see from Fig. 2 that support of the same feature in the two classes are still close.

\section{B. Experiment settings}

\section{1) Prediction models}

We implemented Gradient Boost [11] as our classifier. Gradient boost classifier is a state-of-the-art ensemble learning method that has achieved improved performance in many studies for hospital readmission prediction [12-14].

The C-Motif algorithm proposed by [5] was chosen as one baseline model. The algorithm generates conditional phosphorylation Motifs, a type of discriminative patterns, based on two statistical significance measures, which are defined below.

Global statistical significance of a pattern $p$, denoted as $\operatorname{Sig}\left(p, D_{1}, D_{2}\right)$, is measured by the support odds ratio:

$$
\operatorname{Sig}\left(p, D_{1}, D_{2}\right)=\frac{\frac{\sup \left(p, D_{1}\right)}{1-\sup \left(p, D_{1}\right)}}{\frac{\sup \left(p, D_{2}\right)}{1-\sup \left(p, D_{2}\right)}}
$$

Local statistical significance of a pattern $p$, denoted as $\operatorname{Sig}_{l}\left(p, D_{1}, D_{2}\right)$, is defined below:

$$
\operatorname{Sig}_{l}\left(p, D_{1}, D_{2}\right)=\min _{1 \leq i \leq k} \operatorname{Sig}\left(p, D_{1}\left(p_{i}\right), D_{2}\left(p_{i}\right)\right)
$$

Where $k$ is the size of $p, D_{1}\left(p_{i}\right)$ and $D_{2}\left(p_{i}\right)$ consist of transactions which contain $p$ 's size $k-1$ sub-patterns without the $i^{\text {th }}$ item, in the two classes, respectively.

Compared with the equation (5), our method from equation (3) measures the scale of support difference over the two classes and selects patterns with the relative support difference no less than the defined threshold.

Discriminative patterns generated by our proposed algorithm were evaluated by the performance of readmission prediction in comparison with patterns from C-Motif. Parameters required for C-Motif are the support threshold, the global significance threshold, and the local significance threshold. We set these parameters where they generate the same number of patterns as we do.

Chi-square was chosen as another baseline feature selection because it has been widely applied and showed the best performance in existing methods for readmission prediction [7,9].

All datasets were extremely imbalanced. In order to deal with the imbalanced class problem, SMOTE (Synthetic minority over-sampling technique) which is the most widely applied in the existing literature was implemented. It creates samples of minority class considering k-nearest minority neighbours [15].

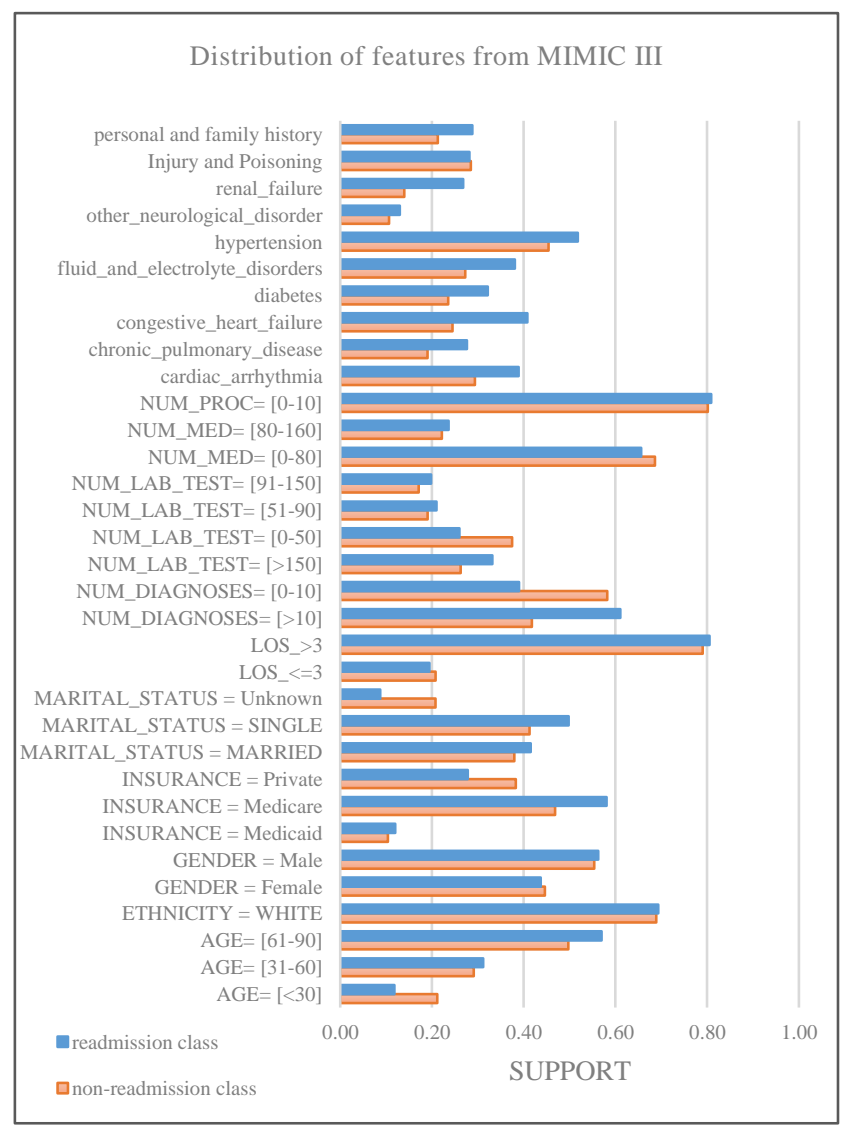

Figure 2 Support of some features in the two classes in MIMIC-III 


\begin{tabular}{|c|c|c|c|}
\hline \multirow[t]{2}{*}{ Features } & \multicolumn{3}{|c|}{ Relative Support Difference (average) } \\
\hline & Diabetes & $C K D . Q L D$ & MIMIC III \\
\hline age & 0.22 & 0.07 & 0.21 \\
\hline ethnicity & - & 0.14 & 0.21 \\
\hline gender & 0.01 & 0.01 & 0.02 \\
\hline insurance & - & - & 0.25 \\
\hline marital status & - & - & 0.28 \\
\hline Length of Stay or Time in hospital & 0.20 & 0.11 & 0.04 \\
\hline Number of emergency visits in the year preceding & 0.21 & - & - \\
\hline Number of inpatient visits for the year preceding & 0.35 & - & - \\
\hline Number of outpatient visits for the year & 0.08 & - & - \\
\hline Number of diagnoses during the admission & 0.18 & 0.18 & 0.32 \\
\hline Number of lab test during the admission & 0.10 & - & 0.19 \\
\hline Number of medications prescribed & 0.13 & - & 0.08 \\
\hline Number of procedures received during the admission & - & 0.03 & 0.11 \\
\hline cardiac arrhythmia & 0.01 & 0.10 & 0.24 \\
\hline chronic pulmonary disease & 0.04 & 0.26 & 0.31 \\
\hline coagulopathy & 0.11 & 0.16 & 0.20 \\
\hline congestive heart failure & 0.15 & 0.31 & 0.40 \\
\hline diabetes & 0.01 & 0.11 & 0.27 \\
\hline fluid and electrolyte disorders & 0.07 & 0.12 & 0.28 \\
\hline hypertension & 0.06 & 0.02 & 0.12 \\
\hline liver disease & - & - & 0.37 \\
\hline pulmonary circulation disorder & - & 0.26 & 0.34 \\
\hline renal failure & 0.23 & 0.12 & 0.48 \\
\hline Persons with a condition influencing their health status & - & 0.11 & 0.48 \\
\hline insulin & 0.08 & - & - \\
\hline metformin & 0.14 & - & - \\
\hline A1C test result & 0.10 & - & - \\
\hline CKD Stages & - & 0.13 & - \\
\hline $\begin{array}{l}\text { Average relative support difference between the readmission class } \\
\text { and non-readmission class }\end{array}$ & 0.15 & 0.11 & 0.27 \\
\hline
\end{tabular}

Table 2 The average of relative support differences of some features used for the readmission prediction. Elixhauser Comorbidities group was used for grouping diagnosis codes.

The prediction model was built in Python using Scikit-learn. Each dataset was split into $70 \%$ for training and $30 \%$ for validation and 10-fold cross-validation was applied.

\section{2) Parameter Settings}

To generate discriminative patterns, we first fixed two parameters, $\theta_{\text {sup }}$ and $w$, as 0.2 and 0.4 , respectively. The weight $w, 0.4$ means we would like to put more weight on relative support difference rather than support when choosing discriminative patterns for the readmission prediction. The threshold of relative difference $\theta_{\text {rsd }}$ was set to $0.3,0.25$, and 0.2 for MIMIC-III, diabetes and CKD.QLD respectively. In order to conduct a fair comparison with C-Motif, we select top 10 discriminative patterns based on the discriminative score.

Hyper-parameters of Gradient Boost Classifier are set to learning_rate $=0.01$, max_depth $=11, \mathrm{n} \_$estimators $=200$.

\section{Evaluation}

\section{1) Discriminative patterns}

Discriminative patterns were generated using Algorithm 1. For the three datasets, the top 10 discriminative patterns were selected based on the discriminative score calculated using Equation (4) for each class. The patterns generated by our model and the C-Motif model are shown in Tables 3, 4, 5 for MIMIC-III, diabetes and CKD.QLD, respectively.

Taking MINIC-III dataset as an example, there are 5 common discriminative patterns from both approaches displayed in bold in Table 3. According to the result, patients who were diagnosed with 'congestive heart failure' and 'diagnoses $=$ [V40-V49]' are likely to be readmitted within 30 days after discharge. The pattern \{'congestive heart failure', 'diagnoses $=$ [V40-V49]' $\}$ is selected as important by both algorithms. Diagnoses codes [V40-V49] is one of chapters of
ICD-9 classification where patients are admitted hospitals with some conditions influencing health status such as mental problems, which can be converted to ICD-10 classification, [Z00-Z99].

The major difference between the proposed algorithm and C-Motif is that C-Motif generates patterns from only one class, i.e. readmission class, while our approach generates patterns from both classes. Therefore, we can find discriminative patterns representing the non-readmission class as well. For example, patients with private insurance, the number of lab test less than 50, and the number of medications less than 10, are unlikely to be readmitted, which is unable to be discovered from C-Motif algorithm. For the readmission class, our model chooses patterns with 'los $=>3$ ' as discriminative, however, C-Motif decides that 'renal failure' is significant. Considering that length of stay is one of the popular features selected by other studies $[1,3,4,7,8,12]$, patterns selected by our method are reasonable.

For diabetes dataset, patterns are displayed in Table 4. Both models consider three patterns: \{number of inpatient visits $=[>=2]$ ' $\},\{$ 'number of inpatient visits $=[>=2]$ ', 'A1Cresult $=$ None' $\}$ and $\{$ 'number of inpatient visits $=[>=2]$ ', 'A1Cresult $=$ None' $\}$ are discriminative. However, our proposed model chooses the pattern $\{$ 'number of diagnoses $=$ $[>=6]$ ', 'number of inpatient visits $=[>=2]$ ' $\}$ is significant while C-Motif selects the pattern \{'number of diagnoses = $[>=6]$ ', 'number of medications $=[>=16]$ ' $\}$ as important. Using our method, we can find that features such as 'number of emergency visits $=[0]$ ', 'number of inpatient visits $=[0-1]$ ', and 'time in hospital $=[0-4]$ ' are highly discriminative for nonreadmission class. 


\begin{tabular}{|c|c|c|}
\hline Methods & Class & Final Discriminative patterns from MIMIC-III \\
\hline \multirow[t]{2}{*}{$\begin{array}{l}\text { Discriminative } \\
\text { patterns }\end{array}$} & Readmission & 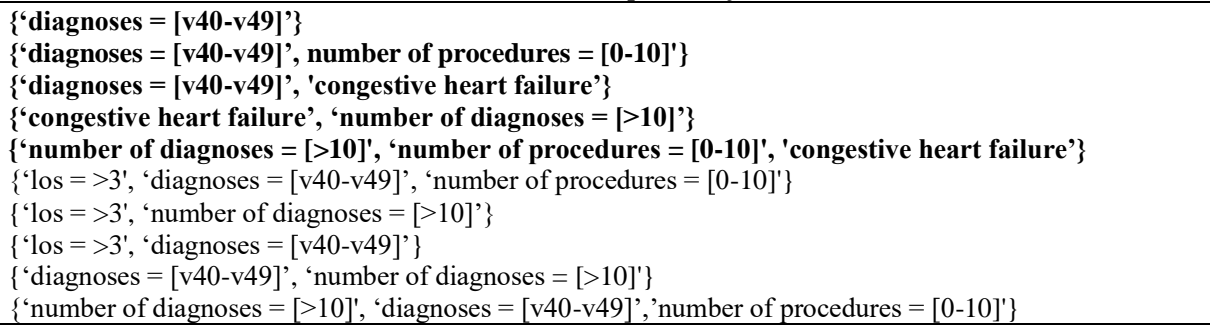 \\
\hline & $\begin{array}{l}\text { Non- } \\
\text { readmission }\end{array}$ & $\begin{array}{l}\{\text { 'marital status }=\text { unknown' }\} \\
\{\text { 'age }=[<30] \text { ' }\} \\
\{\text { 'insurance = private', 'number of lab tests }=[0-50] \text { ' }\} \\
\{\text { 'insurance }=\text { private', 'number of lab tests }=[0-50] \text { ', 'number of medications }=[0-80] '\} \\
\{\text { 'insurance }=\text { private', 'number of lab tests }=[0-50] \text { ', number of procedures }=[0-10] '\} \\
\{\text { 'insurance = private', 'number of diagnoses }=[0-10] '\} \\
\{\text { 'number of diagnoses }=[0-10] \text { ', 'number of lab tests }=[0-50] \text { ', 'number of medications }=[0-80] \text { ' }\} \\
\{\text { 'number of diagnoses }=[0-10] \text { ', 'number of lab tests }=[0-50] '\} \\
\{\text { 'number of diagnoses }=[0-10] \text { ', 'number of lab tests }=[0-50] \text { ', number of procedures }=[0-10] \text { ' }\} \\
\{\text { 'los }=>3 \text { ', 'number of diagnoses }=[0-10] \text { ' }\}\end{array}$ \\
\hline C-Motif & Readmission & 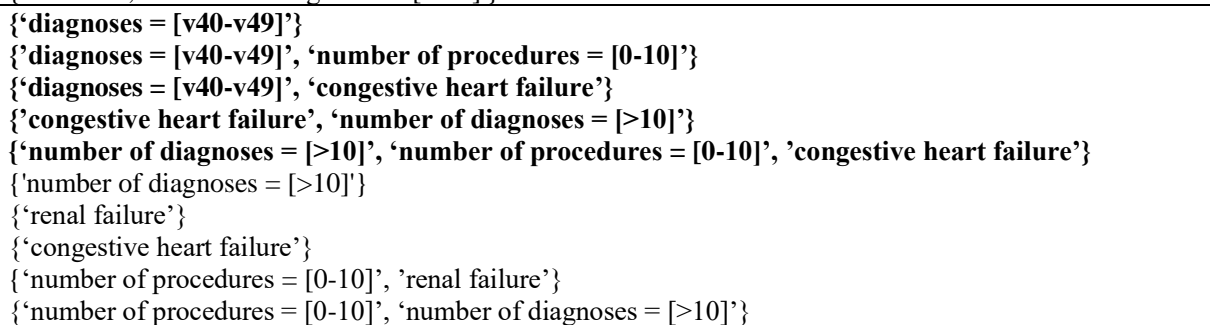 \\
\hline Dataset & Class & Final Discriminative patterns from Diabetes \\
\hline \multirow[t]{2}{*}{$\begin{array}{l}\text { Discriminative } \\
\text { patterns }\end{array}$} & Readmission & $\begin{array}{l}\{\text { A1Cresult }=\text { None', 'number of inpatient visits }=[>=\mathbf{2}] \text { ' }\} \\
\{\text { 'number of inpatient visits }=[>=\mathbf{2}] \text { ' }\} \\
\{\text { 'A1Cresult }=\text { None', 'number of inpatient visits }=[>=2] \text { ', 'glucose serum test }=\text { None' }\} \\
\{\text { 'number of inpatient visits }=[>=2] \text { ', 'glucose serum test }=\text { None' }\} \\
\{\text { 'number of diagnoses }=[>=6] \text { ', 'A1Cresult }=\text { None', 'number of inpatient visits }=[>=2] \text { ' }\} \\
\{\text { 'number of inpatient visits }=[>=2] \text { ', 'race }=\text { Caucasian' }\} \\
\{\text { 'number of diagnoses }=[>=6] \text { ', 'number of inpatient visits }=[>=2] \text { ', 'glucose serum test }=\text { None' }\} \\
\{\text { 'number of diagnoses }=[>=6] \text { ', 'number of inpatient visits }=[>=2] \text { ' }\} \\
\{\text { 'number of diagnoses }=[>=6] \text { ', 'A1Cresult }=\text { None', 'congestive heart failure' }\} \\
\{\text { 'A1Cresult }=\text { None', 'number of inpatient visits }=[>=2] \text { ', 'congestive heart failure' }\}\end{array}$ \\
\hline & $\begin{array}{l}\text { Non- } \\
\text { readmission }\end{array}$ & $\begin{array}{l}\{\text { 'number of inpatient visits }=[0-1] \text { ', 'time in hospital }=[0-4] \text { ' }\} \\
\{\text { 'number of emergency visits }=[0] \text { ', 'number of inpatient visits }=[0-1] \text { ', 'time in hospital }=[0-4] \text { ' }\} \\
\{\text { 'number of inpatient visits }=[0-1] \text { ', 'time in hospital }=[0-4] \text { ', ' } \text { glucose serum test }=\text { None' }\} \\
\{\text { 'number of inpatient visits }=[0-1] \text { ', 'number of medications }=[0-15] \text { ' }\} \\
\{\text { 'number of outpatient visits }=[0] \text { ', 'number of inpatient visits }=[0-1] \text { ', 'time in hospital }=[0-4] \text { ' }\} \\
\{\text { 'number of emergency visits }=[0] \text { ', 'number of medications }=[0-15] \text { ', 'number of inpatient visits }=[0-1] \text { ' }\} \\
\{\text { 'number of inpatient visits }=[0-1] \text { ', 'number of medications }=[0-15] \text { ', 'time in hospital }=[0-4]\} \\
\{\text { 'number of outpatient visits }=[0] \text { ', 'number of medications }=[0-15] \text { ', 'number of inpatient visits }=[0-1] \text { ' }\} \\
\{\text { 'number of lab procedures }=[0-50] \text { ', 'number of inpatient visits }=[0-1] \text { ', 'time in hospital }=[0-4] \text { ' }\} \\
\left\{\text { 'number of emergency visits }=[0] \text { ', 'number of inpatient visits }=[0-1] \text { ', 'age }=[30-60)^{\prime}\right\}\end{array}$ \\
\hline C-Motif & Readmission & $\begin{array}{l}\{\text { 'number of inpatient visits }=[>=2] \text { ' } \\
\{\text { 'number of diagnoses }=[>=6] '\} \\
\{\text { 'number of inpatient visits }=[>=2] \text { ', 'A1Cresult }=\text { None' }\} \\
\{\text { 'A1Cresult = None', 'congestive heart failure' }\} \\
\{\text { 'number of diagnoses }=[>=6] \text { ', 'A1Cresult }=\text { None' }\} \\
\{\text { 'number of diagnoses }=[>=6] \text { ', 'number of medications }=[>=16] '\} \\
\{\text { 'number of diagnoses }=[>=6] \text { ', 'number of medications }=[>=16] \text { ', 'A1Cresult = None' }\} \\
\{\text { 'insulin', 'number of diagnoses }=[>=6] \text { ', 'A1Cresult }=\text { None' }\} \\
\{\text { 'number of diagnoses }=[>=6] \text { ', 'A1Cresult }=\text { None', 'congestive heart failure' }\} \\
\{\text { 'race = Caucasian', 'insulin', 'A1Cresult = None' }\}\end{array}$ \\
\hline
\end{tabular}




\begin{tabular}{|c|c|c|}
\hline Dataset & Class & Final Discriminative patterns from CKD.QLD \\
\hline \multirow[t]{2}{*}{$\begin{array}{l}\text { Discriminative } \\
\text { Patterns }\end{array}$} & Readmission & 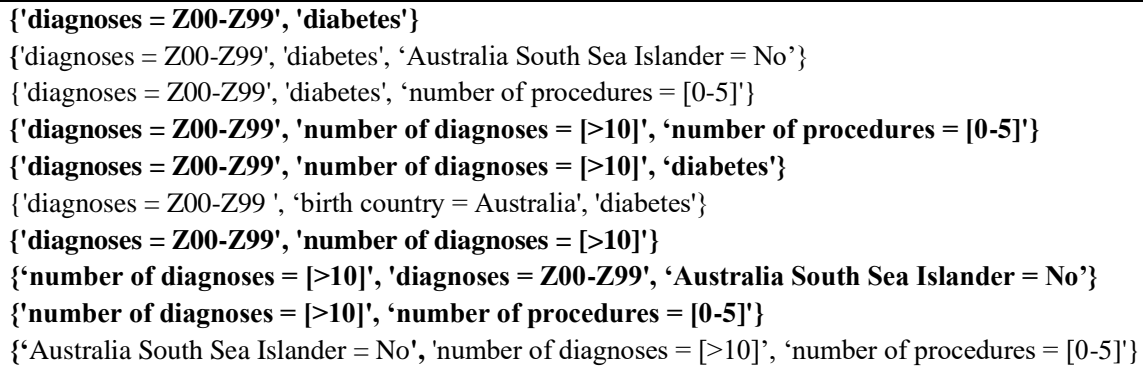 \\
\hline & $\begin{array}{l}\text { Non- } \\
\text { readmission }\end{array}$ & $\begin{array}{l}\{\text { 'number of diagnoses }=[0-10] \text { ', 'ckd stage }=\text { No', 'sex }=\text { male' }\} \\
\{\text { 'diagnoses = U00-U99', 'number of diagnoses }=[0-10] \text { ', 'Indigenous status = Not ' }\} \\
\{\text { 'diagnoses = U00-U99', 'los_<=4', 'number of diagnoses }=[0-10] \text { ' }\} \\
\{\text { 'los_<=4', 'number of diagnoses }=[0-10] \text { ', 'ckd stage }=\text { No' }\} \\
\{\text { 'Indigenous status = Not ', 'age }=[31-60] \text { ', 'ckd stage }=\text { No' }\}\end{array}$ \\
\hline C-Motif & Readmission & 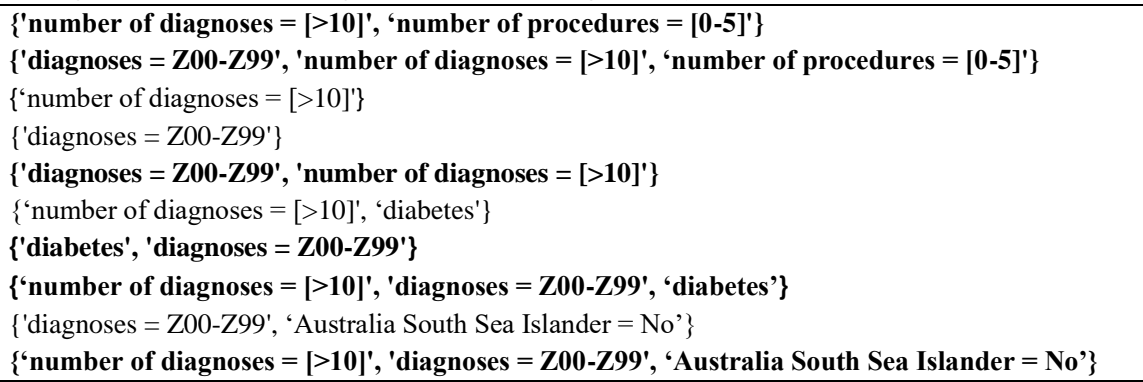 \\
\hline
\end{tabular}

Table 5 Comparison of the patterns generated by our proposed algorithm and C-Motif from CKD.QLD dataset. Patterns in Bold indicates that they were discriminative from both methods.

From Table 5, for chronic kidney disease patients, our method decides the pattern \{'diabetes', 'diagnoses $=[\mathrm{Z} 00$ Z99]' $\}$ as discriminative whereas C-Motif selects the pattern and all subsets of the pattern, $\{$ 'diabetes' $\}$ and $\{$ 'diagnoses $=$ [Z00-Z99]'\}, as important. Additionally, our method suggests that patterns $\{$ 'diagnoses $=\mathrm{U} 00-\mathrm{U} 99$ ', 'number of diagnoses $=$ [0-10]', 'Indigenous status = Not ' $\},\{$ 'diagnoses = U00-U99', 'los_<=4', 'number of diagnoses $=[0-10]$ ' $\}$ are closely related to non-readmission class.

From the patterns generated for all the three datasets, we can see that age, prior hospital utilisation, length of stay, and the number of diagnoses are important factors that differentiate the two classes, which is consistent with results from the literature [20]. Comorbidities such as 'congestive heart failure', 'diabetes' and 'diagnoses $=[\mathrm{V} 40-\mathrm{V} 99]$ or [Z00Z99]' are also significant predictors for readmissions.

\section{2) Readmission Prediction evaluation}

We evaluated the performance of readmission prediction models with the proposed discriminative patterns as features in terms of Area Under the receiver operating characteristics Curve (AUC).

Table 6 shows that pattern-based features mined by both our algorithm and C-Motif perform better than the model with individual features. Our proposed model with MIMIC III presents $12 \%$ of improvement over chi-square selection. However, discriminative patterns from both the diabetes and CKD.QLD datasets do not significantly improve the prediction performance. This is because there exists little support for the differences in features between the readmission class and the non-readmission class in these two datasets so that even discriminative patterns mined by our algorithm hardly differentiate the two classes.

\begin{tabular}{|l|c|c|c|}
\hline & Diabetes & MIMIC-III & CKD.QLD \\
\hline Chi-Square & 0.53 & 0.55 & 0.53 \\
\hline C-Motif & 0.57 & 0.62 & 0.53 \\
\hline Proposed model & 0.58 & 0.62 & 0.54 \\
\hline
\end{tabular}

Table 6 The area under the receiver operating characteristics curve (AUC) of the Gradient Boost classifier built with patterns generated by C-Motif and Our proposed algorithm.

\section{CONCLUSION}

In this research, we proposed the discriminative patterns to achieve useful insights and improve the prediction performance of 30-days hospital readmission. Currently, feature selection methods proposed by the literature only consider the importance of single feature to the readmission outcome, showing moderate results. Discriminative patterns are selected concerning the support differences and frequency of patterns between the readmission class and the noreadmission class. The proposed models outperformed the baseline, gradient boost model with chi-square selection, showing that pattern-based features enhance the performance of readmission prediction. However, the improvement was not significant. From the experiments, because patients have similar patterns either readmitted or not, traditional classification methods cannot predict readmission accurately and therefore, more sophistic algorithms such as deep learning which can find latent relationships between features are required.

We acknowledge the limitations of our study. Firstly, we used only diagnosis codes for medical information. However, there exist more information such as procedures, and medications prescribed. More detailed information can improve the performance of readmission prediction. Secondly, sequences have not been taken into consideration in this study. It is natural especially for patients with chronic diseases to have multiple admissions. From the results of our 
experiments, features regarding hospital utilisation have significant roles in predicting readmission. Some diseases or treatments from each admission could be a primary cause for the next admission. Therefore, deep learning algorithms will be developed which considers the history of patient hospital utilisation to predict 30-day readmission accurately. Lastly, deep learning-based models need a very large number of training samples to accurately generate the relationships between features. Fortunately, in medical areas, there are wellestablished domain ontologies which describe relationships between medical concepts by hierarchical structure such as ICD-9 and CCS (Clinical Classification Software). Our future work will utilize the hierarchical relationships among medical concepts in medical ontologies to improve the performance of readmission prediction.

\section{ACKNOWLEDGMENT}

We thank the NHMRC Chronic Kidney Disease Centre of Research Excellence and Chronic Kidney Disease in Queensland for providing the CKD.QLD datasets supported by NHMRC grant (No. APP1079502) with ethics approval (No. HERC/15/QRBW/294).

\section{REFERENCES}

[1] Zheng B, Zhang J, Yoon SW, Lam SS, Khasawneh M, Poranki S. Predictive modeling of hospital readmissions using metaheuristics and data mining. Expert Systems with Applications. 2015;42(20):7110-20.

[2] Amarasingham R, Moore BJ, Tabak YP, Drazner MH, Clark CA, Zhang S, et al. An automated model to identify heart failure patients at risk for 30-day readmission or death using electronic medical record data. Medical Care. 2010;48(11):981-8

[3] Au AG, McAlister FA, Bakal JA, Ezekowitz J, Kaul P, van Walraven C. Predicting the risk of unplanned readmission or death within 30 days of discharge after a heart failure hospitalization. American Heart Journal. 2012;164(3):365-72.

[4] Futoma J, Morris J, Lucas J. A comparison of models for predicting early hospital readmissions. Journal of Biomedical Informatics. 2015;56:229-38.

[5] Liu X, Wu J, Gu F, et al. Mining conditional phosphorylation motifs. IEEE/ACM Trans. Comput. Biol Bioinform 2014; 11(5).

[6] van Walraven C, Dhalla IA, Bell C, Etchells E, Stiell IG, Zarnke K, et al. Derivation and validation of an index to predict early death or unplanned readmission after discharge from hospital to the community. Can Med Assoc J. 2010;182(6):551-7.

[7] Collins J, Abbassa IM, Harveya R, Suehsa B, Uribea C, Bouchardb J, et al. Predictors of all-cause 30 day readmission among Medicare patients with type 2 diabetes. Current Medical Research and Opinion. 2017;33(8):1517-23.

[8] Bradford C, Shah BM, Shane P, Wachi N, Sahota K. Patient and clinical characteristics that heighten risk for heart failure readmission. Research in Social and Administrative Pharmacy. 2017;13(6):1070-81.

[9] Cui S, Wang D, Wang Y, Yu P-W, Jin Y. An improved support vector machine-based diabetic readmission prediction. Computer Methods and Programs in Biomedicine. 2018;166:123-35

[10] Cheng H, Yan X, Han J, Yu PS, editors. Direct Discriminative Pattern Mining for Effective Classification. 2008 IEEE 24th International Conference on Data Engineering; 2008 7-12 April 2008.

[11] Friedman JH. Greedy Function Approximation: A Gradient Boosting Machine. The Annals of Statistics. 2001;29(5):1189-232.

[12] Maali Y, Perez-Concha O, Coiera E, Roffe D, Day RO, Gallego B. Predicting 7-day, 30-day and 60-day all-cause unplanned readmission: a case study of a Sydney hospital. BMC Med Inform Decis Mak. 2018;18(1):1

[13] Min X, Yu B, Wang F, Predictive Modeling of the Hospital Readmission Risk from Patients' Claims Data Using Machine Learning: A Case Study on COPD. Sci Rep-Uk. 2019;9(1):2362.
[14] Rahimian F, Salimi-Khorshidi G, Payberah AH, Tran J, Ayala Solares $\mathrm{R}$, Raimondi $\mathrm{F}$, et al. Predicting the risk of emergency admission with machine learning: Development and validation using linked electronic health records. PLOS Medicine. 2018;15(11):e1002695.

[15] Chawla N, Bowyer K, Hall L, Kegelmeyer W. SMOTE: Synthetic Minority Over-sampling Technique. arXivorg. 2011;16.

[16] Strack B, DeShazo JP, Gennings C, Olmo JL, Ventura S, Cios KJ, et al. Impact of $\mathrm{HbA} 1 \mathrm{c}$ measurement on hospital readmission rates: analysis of 70,000 clinical database patient records. BioMed Research International. 2014;2014:781670.

[17] Goldberger Ary L, Amaral Luis AN, Glass L, Hausdorff Jeffrey M, Ivanov Plamen C, Mark Roger G, et al. PhysioBank, PhysioToolkit, and PhysioNet Components of a New Research Resource for Complex Physiologic Signals. Circulation. 2000;101(23):e215-e20.

[18] Venuthurupalli SK, Hoy WE, Healy HG, Cameron A, Fassett RG. CKD.QLD: establishment of a chronic kidney disease [CKD] registry in Queensland, Australia. Bmc Nephrol. 2017;18(1):189.

[19] Elixhauser RA, Steiner MC, Harris MD, Coffey MR. Comorbidity Measures for Use with Administrative Data. Medical Care. 1998;36(1):8-27.

[20] Zhou H, Della PR, Roberts P, Goh L, Dhaliwal SS. Utility of models to predict 28-day or 30-day unplanned hospital readmissions: an updated systematic review. BMJ Open. 2016;6(6):e011060.

[21] By SD, Pazzani MJ. Detecting group differences: mining contrast sets. Data Mining Knowledge Discover, 2001; 5(3).

[22] Fang G, Pandey G, Wang W, et al. Mining low-support discriminative patterns from dense and high-dimensional data. IEEE Transactions on Knowledge Data Engineering, 2012, 24(2).

[23] HCUP Elixhauser Comorbidity Software. Healthcare Cost and Utilization Project (HCUP). June 2017. Agency for Healthcare Research and Quality, Rockville, MD. www.hcupus.ahrq.gov/toolssoftware/comorbidity/comorbidity.jsp.

[24] Australian Institute of Health and Welfare. Chronic kidney disease [Internet]. Canberra: Australian Institute of Health and Welfare, 2020 [cited 2020 Aug. 17]. Available from: https://www.aihw.gov.au/reports/chronic-kidney-disease/chronickidney-disease 